\title{
RECURSIVE FILTERING FOR SPLINES ON HEXAGONAL LATTICES
}

\author{
Dimitri Van De Ville, Thierry Blu, Michael Unser \\ Biomedical Imaging Group, STI/BIO-E \\ Swiss Federal Institute of Technology Lausanne (EPFL) \\ CH-1015 Lausanne EPFL, Switzerland
}

\begin{abstract}
Hex-splines are a novel family of bivariate splines, which are well suited to handle hexagonally sampled data. Similar to classical 1D B-splines, the spline coefficients need to be computed by a prefilter. Unfortunately, the elegant implementation of this prefilter by causal and anti-causal recursive filtering is not applicable for the (non-separable) hex-splines. Therefore, in this paper we introduce a novel approach from the viewpoint of approximation theory. We propose three different recursive filters and optimize their parameters such that a desired order of approximation is obtained. The results for third and fourth order hex-splines are discussed. Although the proposed solutions provide only quasi-interpolation, they tend to be very close to the interpolation prefilter.
\end{abstract}

\section{INTRODUCTION}

B-splines have shown to be of great importance for signal and image processing. In particular, they provide an essential link between the discrete and the continuous domain which is required for many fundamental operations such as interpolation and resampling [1]. Recently, we introduced a novel family of splines, called hex-splines. Derived from the shape of the Voronoi cell of a hexagonal lattice, they preserve the twelve-fold symmetry of the hexagon [2]. These bivariate splines, different from box-splines [3], exhibit many properties similar to the B-splines.

An important operation in spline processing is the computation of the spline coefficients, which projects a given signal into the spline space. In practice, this requires prefiltering of the signal sample values. In this paper, we discuss this prefiltering step, sometimes also called the direct spline transform, for the case of hex-splines. One way the filter operation can be implemented is in the Fourier domain. Unfortunately, as opposed to separable B-splines, there is no exact implementation in the spatial domain using recursive filtering. Nevertheless, we propose here to use recursive filters anyway. Our method is inspired by the 1D $\mathrm{B}$-spline case and is optimized to approach the orthogonal projection into the hex-spline space as close as possible. (a)

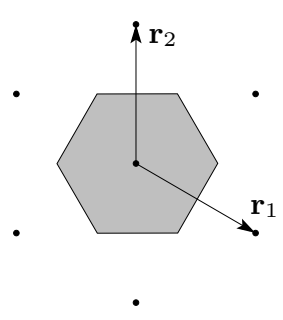

Fig. 1. (a) The regular hexagonal lattice of the second type and its Voronoi cell. (b) The dual lattice.

\section{SPLINES FOR HEXAGONAL LATTICES}

\subsection{Fundamental properties}

A $2 \mathrm{D}$ periodic lattice is described by two vectors $\mathbf{r}_{1}$ and $\mathbf{r}_{2}$. It is convenient to group these vectors in a lattice matrix $\mathbf{R}=\left[\mathbf{r}_{1} \mid \mathbf{r}_{2}\right]$. A well-known unique tiling cell is the Voronoi cell, which contains all points closer to their lattice site than to any other site. The reciprocal or dual lattice corresponds to the matrix $\hat{\mathbf{R}}=\left[\hat{\mathbf{r}}_{1} \mid \hat{\mathbf{r}}_{2}\right]=\left(\mathbf{R}^{-1}\right)^{\mathrm{T}}=\mathbf{R}^{-\mathrm{T}}$. As an example, Fig. 1 shows the regular hexagonal lattice of the second type with lattice matrices:

$$
\mathbf{R}=\left[\begin{array}{cc}
\frac{\sqrt{3}}{2} & 0 \\
-\frac{1}{2} & 1
\end{array}\right], \quad \hat{\mathbf{R}}=\left[\begin{array}{cc}
\frac{2 \sqrt{3}}{3} & \frac{\sqrt{3}}{3} \\
0 & 1
\end{array}\right] .
$$

The areas colored in gray correspond to the Voronoi cells. More details can be found in [4].

Hex-splines are now defined as follows. The first-order hex-spline $\eta_{1}(\mathbf{x})$ is the indicator function of the Voronoi cell. Higher order hex-splines are constructed by successive convolutions:

$$
\eta_{p}(\mathbf{x})=\frac{\eta_{1} \otimes \eta_{p-1}(\mathbf{x})}{\Omega}, \quad p>1
$$

normalized by the surface area of the Voronoi cell $\Omega \triangleq$ $|\operatorname{det}(\mathbf{R})|$. The signal space spanned by the hex-splines con- 
tains all signals

$$
s(\mathbf{x})=\sum_{\mathbf{k}} c(\mathbf{k}) \eta_{p}(\mathbf{x}-\mathbf{R} \mathbf{k}) ; c(\mathbf{k}) \in l_{2}\left(\mathbb{Z}^{2}\right)
$$

In general, the coefficients $c(\mathbf{k})$ are determined as

$$
c(\mathbf{k})=\int g(\mathbf{x}) \tilde{\varphi}(\mathbf{x}-\mathbf{R} \mathbf{k}) \mathbf{d} \mathbf{x},
$$

where $\tilde{\varphi}$ is the prefilter. The optimal choice, i.e., corresponding to an orthogonal projection into the spline space, is the dual filter $\hat{\tilde{\varphi}}_{d}=\hat{\varphi} / \hat{a}_{\varphi}$. Here $\hat{a}_{\varphi}$ is the Fourier transform of the sampled autocorrelation function. In particular for the hex-splines, we have

$$
a_{\varphi}(\mathbf{k})=\left\langle\eta_{p}(\mathbf{x}), \eta_{p}(\mathbf{x}-\mathbf{R k})\right\rangle=\Omega \eta_{2 p}(\mathbf{R k}) .
$$

Another popular choice for $\tilde{\varphi}$ is the interpolation prefilter, which makes $s(\mathbf{R k})=g(\mathbf{R k})$ at the sampling sites. For the first and second order splines, this condition is trivially satisfied by choosing $c(\mathbf{k})=g(\mathbf{R k})$. But for higher orders, as in the classical case, one needs a prefilter (which only uses sample values of the original signal) to obtain the correct values for $c(\mathbf{k})$. In general, the interpolating condition can be written as

$$
\begin{aligned}
g(\mathbf{R} \mathbf{l}) & =\sum_{\mathbf{k}} c(\mathbf{k}) \eta_{p}(\mathbf{R} \mathbf{l}-\mathbf{R} \mathbf{k}) \\
& =\sum_{\mathbf{k}} c(\mathbf{k}) h_{p}(\mathbf{l}-\mathbf{k})
\end{aligned}
$$

where we introduce the discrete hex-spline as $h_{p}(\mathbf{k})=\eta_{p}(\mathbf{R k})$. In order to obtain $c(\mathbf{k})$ out of Eq. (6), we need to filter the sample values with the inverse filter $h_{p}^{-1}$. To specify the filter, we introduce the $\mathcal{Z}$-transform

$$
\check{f}(\mathbf{z})=\sum_{\mathbf{k}} \mathbf{z}^{-\mathbf{k}} f(\mathbf{R} \mathbf{k}),
$$

where we use the notation $\mathbf{z}^{\mathbf{k}}=z_{1}^{k_{1}} z_{2}^{k_{2}}$. Then, the Fourier transform of the sampled version $f(\mathbf{R k})$ is

$$
\hat{f}_{\mathbf{R}}(\boldsymbol{\omega})=\check{f}\left(\exp \left(j \mathbf{R}^{\mathrm{T}} \boldsymbol{\omega}\right)\right) .
$$

As an example, the $\mathcal{Z}$-transform of the third and fourth order discrete hex-splines can be computed as (we make use of $z_{3}=z_{1} z_{2}$ )

$$
\begin{aligned}
& \check{h}_{3}\left(z_{1}, z_{2}\right)=\frac{42}{72}+\frac{5}{72}\left(z_{1}+z_{2}+z_{1}^{-1}+z_{2}^{-1}+z_{3}+z_{3}^{-1}\right) \\
& \check{h}_{4}\left(z_{1}, z_{2}\right)= \\
& \quad \frac{37}{81}+\frac{29}{324}\left(z_{1}+z_{2}+z_{1}^{-1}+z_{2}^{-1}+z_{3}+z_{3}^{-1}\right)+ \\
& \quad \frac{1}{972}\left(z_{1}^{-1} z_{2}+z_{1} z_{2}^{-1}+z_{1} z_{2}^{2}+z_{1}^{-1} z_{2}^{-2}+z_{3}^{2}+z_{3}^{-2}\right) .
\end{aligned}
$$

Computing the spline coefficients is performed by filtering the sample values by $\tilde{\varphi}=\left(h_{p}\right)^{-1}$. This particular filter is called the interpolation prefilter.

\subsection{Approximation theory}

Approximation theory provides us with a convenient way to quantify the approximation error by integration with an error kernel $E(\boldsymbol{\omega})$ in the Fourier domain: [5]

$$
\|s(\mathbf{x})-g(\mathbf{x})\|^{2}=\frac{1}{4 \pi^{2}} \int|\hat{g}(\boldsymbol{\omega})|^{2} E(\boldsymbol{\omega}) \mathrm{d} \boldsymbol{\omega} .
$$

The error kernel [5] can be easily extended for a periodic lattice with matrix $\mathbf{R}$ as

$$
E(\boldsymbol{\omega})=\underbrace{1-\frac{|\hat{\varphi}(\boldsymbol{\omega})|^{2}}{\hat{a}_{\varphi}(\boldsymbol{\omega})}}_{E_{\mathrm{min}}}+\underbrace{\frac{\hat{a}_{\varphi}(\boldsymbol{\omega})}{\Omega}\left|\hat{\tilde{\varphi}}(\boldsymbol{\omega})-\hat{\tilde{\varphi}}_{d}(\boldsymbol{\omega})\right|^{2}}_{E_{\mathrm{res}}},
$$

where $\varphi$ is the reconstruction function (in our case $\varphi=$ $\left.\eta_{p}\right), \tilde{\varphi}$ is the prefilter, $\tilde{\varphi}_{d}$ is the optimal prefilter, and $a_{\varphi}$ the sampled autocorrelation function. In the case of using the optimal prefilter, the kernel reduces to $E_{\min }$. When the sampling lattice is made finer by a scaling factor $T$, the error decreases as

$$
\|s(\mathbf{x})-g(\mathbf{x})\|^{2} \propto T^{2 L}
$$

where $L$ is the order of approximation. This behavior can be analyzed by examining the error kernel $E(\boldsymbol{\omega})$ around $\mathbf{0 .}$ Note that the hex-spline $\eta_{p}$ has $p$-th order of approximation.

\section{COMPUTATION OF THE SPLINE COEFFICIENTS}

For this paper we concentrate on the third $(p=3)$ and fourth $(p=4)$ order hex-splines, which are relevant for most applications.

\subsection{Interpolation prefilter}

The interpolation prefilter cannot be implemented exactly in the spatial domain since no decomposition in causal and anti-causal filters is available. A viable solution is to filter the sample values in the Fourier domain by the response $1 / \hat{h}_{3}(\boldsymbol{\omega})$ or $1 / \hat{h}_{4}(\boldsymbol{\omega})$. This can be done by adapting the FFT algorithm for the hexagonal lattice [6,7]. For more details about this approach, see [8].

Figure 2 shows the error kernel corresponding to the interpolation prefilter. We observe that the Nyquist area (i.e., the reciprocal Voronoi cell) appears. Inside, all frequency components are well preserved. Outside, the error tends to 2 , as can be expected $[1,5]$.

\subsection{Recursive prefilter}

For the 1D B-spline case, an elegant approach exists based on causal and anti-causal recursive filtering [9]. Inspired by the $1 \mathrm{D}$ case, we first define the following basic filter operation in the $\mathcal{Z}$-domain: $H(z)=1 /\left(\left(1+\alpha_{0} z\right)\left(1+\alpha_{0} z^{-1}\right)\right)$. 


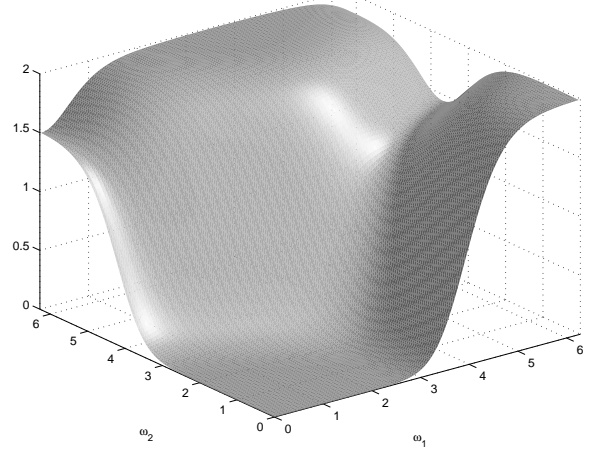

Fig. 2. The error kernel $E(\boldsymbol{\omega})$ corresponding to the fourthorder interpolation prefilter.

Table 1. Recursive filter designs on the hexagonal lattice

\begin{tabular}{lllll}
\hline Filter & Directions & $p=3$ & $p=4$ \\
\hline$\tilde{h}_{1}$ & & ok & - \\
$\tilde{h}_{2}$ & & ok & ok \\
$\tilde{h}_{3}$ & & ok & ok \\
\hline
\end{tabular}

Next, we propose three plausible filter designs on the hexagonal lattice: (see Tab. 1)

$\check{\tilde{h}}_{1}(\mathbf{z})=\alpha_{1}\left(H\left(z_{1}\right)+H\left(z_{2}\right)+H\left(z_{3}\right)\right)$,

$\check{\tilde{h}}_{2}(\mathbf{z})=\alpha_{1}\left(H\left(z_{1}\right) H\left(z_{2}\right)+H\left(z_{1}\right) B\left(z_{3}\right)+H\left(z_{2}\right) H\left(z_{3}\right)\right)$,

$\check{\tilde{h}}_{3}(\mathbf{z})=\alpha_{1} H\left(z_{1}\right) H\left(z_{2}\right) H\left(z_{3}\right)$.

To find the best parameters $\alpha_{0}$ and $\alpha_{1}$ for each proposed filter, we require the order of approximation to be the same as for the optimal prefilter, i.e., 3 and 4 . Considering the error kernel, this can be done by putting a constraint on the second term $E_{\text {res: }}$ :

$$
\hat{\tilde{\varphi}}(\boldsymbol{\omega})-\hat{\tilde{\varphi}}_{d}(\boldsymbol{\omega})=O\left(\|\boldsymbol{\omega}\|^{4}\right)
$$

By calculating the multivariate Taylor series, we obtain

$$
\begin{aligned}
& \frac{\hat{\eta}_{3, d}(\boldsymbol{\omega})}{\Omega}=1+\frac{5}{48}\left(\omega_{1}^{2}+\omega_{2}^{2}\right)+O\left(\|\boldsymbol{\omega}\|^{4}\right), \\
& \frac{\hat{\eta}_{4, d}(\boldsymbol{\omega})}{\Omega}=1+\frac{5}{36}\left(\omega_{1}^{2}+\omega_{2}^{2}\right)+O\left(\|\boldsymbol{\omega}\|^{4}\right),
\end{aligned}
$$

and

$$
\begin{aligned}
& \hat{\tilde{h}}_{1}(\boldsymbol{\omega})=\frac{3 \alpha_{1}}{\left(1+\alpha_{0}\right)^{2}}+\frac{3 \alpha_{0} \alpha_{1}}{2\left(1+\alpha_{0}\right)^{4}}\left(\omega_{1}^{2}+\omega_{2}^{2}\right)+O\left(\|\boldsymbol{\omega}\|^{4}\right), \\
& \hat{\tilde{h}}_{2}(\boldsymbol{\omega})=\frac{3 \alpha_{1}}{\left(1+\alpha_{0}\right)^{4}}+\frac{3 \alpha_{0} \alpha_{1}}{\left(1+\alpha_{0}\right)^{6}}\left(\omega_{1}^{2}+\omega_{2}^{2}\right)+O\left(\|\boldsymbol{\omega}\|^{4}\right), \\
& \hat{\tilde{h}}_{3}(\boldsymbol{\omega})=\frac{\alpha_{1}}{\left(1+\alpha_{0}\right)^{6}}+\frac{3 \alpha_{0} \alpha_{1}}{2\left(1+\alpha_{0}\right)^{8}}\left(\omega_{1}^{2}+\omega_{2}^{2}\right)+O\left(\|\boldsymbol{\omega}\|^{4}\right) .
\end{aligned}
$$

Now we impose the order of approximation condition of Eq. (10). For the third order $(p=3)$, we find a solution for each proposed filter:

$$
\begin{array}{lll}
\tilde{h}_{1}: & \alpha_{0}=\frac{7-2 \sqrt{6}}{5}, \quad \alpha_{1}=\frac{56-16 \sqrt{6}}{25}, \\
\tilde{h}_{2}: & \alpha_{0}=\frac{19-4 \sqrt{21}}{5}, \quad \alpha_{1}=\frac{535296-116736 \sqrt{21}}{625}, \\
\tilde{h}_{3}: & \alpha_{0}=\frac{31-6 \sqrt{26}}{5}, & \alpha_{1}=\frac{43609923072-8552604672 \sqrt{26}}{15625} .
\end{array}
$$

However, for the fourth order, the first filter has no solution:

$$
\begin{array}{ll}
\tilde{h}_{1}: & \text { no solution, } \\
\tilde{h}_{2}: & \alpha_{0}=\frac{1}{5}, \quad \alpha_{1}=\frac{432}{625}, \\
\tilde{h}_{3}: & \alpha_{0}=\frac{22-3 \sqrt{5}}{5}, \quad \alpha_{1}=\frac{6446891088-902741112 \sqrt{51}}{15625} .
\end{array}
$$

Finally, the implementation of these filters is similar to the $1 \mathrm{D}$ implementation of the $H(z)$ filter for classical Bsplines [9]. Here, we also use mirror boundary conditions.

\subsection{Results}

One important remark about the proposed prefilters is that they are only quasi-interpolants; i.e., they will generally not yield an exact interpolation of the signal unless it is a polynomial up to degree $p-1$ in $x_{1}$ or $x_{2}$. Nevertheless, the error kernel of each of these filters closely resembles by design the one of the orthogonal projection (i.e., of the optimal prefilter $\tilde{\varphi}_{d}$ ). In fact, the only differences are near the border and outside of the Nyquist area, where we observe small fluctuations up to $10 \%$ (which have a minor influence on the result).

Now we want to compare the asymptotic constants for each of the filter solutions. These constants allow us to compare the behavior of $E(\boldsymbol{\omega})$ for $\boldsymbol{\omega} \rightarrow \mathbf{0}$ between different solutions when the order of approximation is the same.

For the third order, the error kernel reduces to

$$
E(\boldsymbol{\omega})=O\left(\|\boldsymbol{\omega}\|^{6}\right)+O\left(\|\boldsymbol{\omega}\|^{8}\right) .
$$

Therefore, the asymptotic behavior only depends on the reconstruction function (i.e., $\left.E_{\min }(\boldsymbol{\omega})\right)$ and not on the proposed filters, as long as they satisfy the design constraint.

For the fourth order, both terms of $E(\boldsymbol{\omega})$ are of $O\left(\|\boldsymbol{\omega}\|^{8}\right)$. After calculation, we obtain the following determining asymptotic behavior of $E_{\text {res }}(\boldsymbol{\omega})$ :

$$
\begin{aligned}
& \tilde{h}_{2}:\left(6 \cdot 10^{-4}\left(\omega_{1}^{4}+\omega_{2}^{4}\right)+10^{-2} \omega_{1}^{2} \omega_{2}^{2}\right)^{2}, \\
& \tilde{h}_{3}:\left(10^{-2}\left(\omega_{1}^{4}+\omega_{2}^{4}\right)+2 \cdot 10^{-2} \omega_{1}^{2} \omega_{2}^{2}\right)^{2} .
\end{aligned}
$$

This makes the second prefilter the best solution from an approximation point of view. Figure 4 shows the impulse response of this quasi-interpolant, i.e., prefilter and reconstruction function together as they are conceptually applied to the sampled data. The function value at $\mathbf{0}$ equals 0.883 , compared to 0.802 for the third proposed filter. 


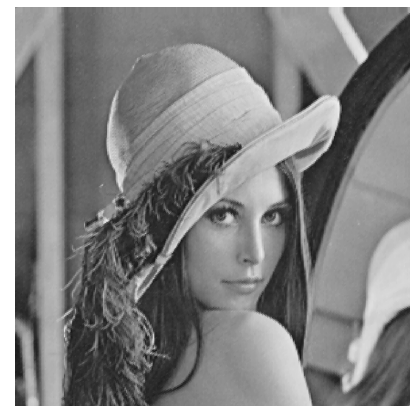

(a)

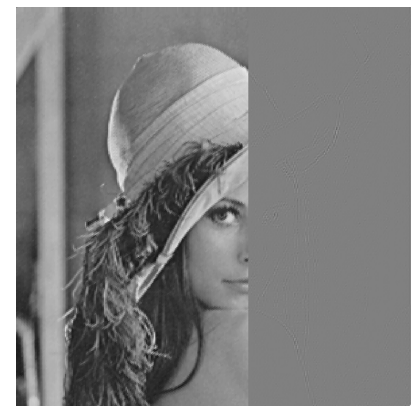

(b)

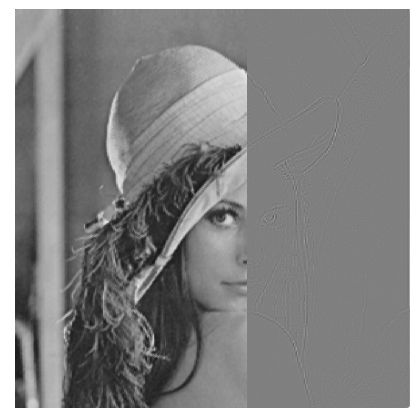

(c)

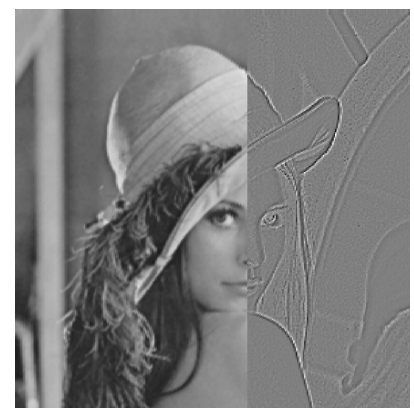

(d)

Fig. 3. The results of the test image "lena" for the fourth-order hex-spline using different prefilters. (a) Prefiltering in the Fourier domain with the interpolation prefilter $1 / \hat{h}^{3}$. (b) Prefiltering in the spatial domain with the recursive filter $\tilde{h}_{2}$. (c) Prefiltering in the spatial domain with $\tilde{h}_{3}$. (d) Without prefilter.

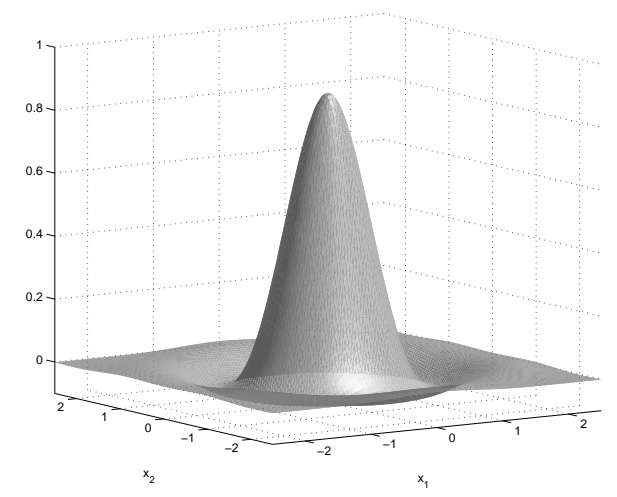

Fig. 4. Impulse response of the combined prefilter and reconstruction function for fourth order: $\left(\eta_{4} \otimes \tilde{h}_{2}\right)(\mathbf{x})$.

Each of the proposed recursive filters has been implemented in the spatial domain. We show the results for the fourth-order hex-spline and the test image "lena", which was first resampled to the hexagonal lattice using cubic Bspline interpolation. Figure 3 (a) shows the result after using the interpolation prefilter with the Fourier method. In (b) and (c), we show the images after spatial recursive filtering by respectively $\tilde{h}_{2}$ and $\tilde{h}_{3}$. The right-hand side of the split shows the difference with (a), multiplied by a factor 4 to increase the visibility. In correspondence with the theoretical results, we observe that the error at the edges is smaller for the second prefilter. Finally, (d) shows the result of a naive approach without prefiltering (i.e., $c(\mathbf{k})=g(\mathbf{R k})$ ) which is clearly not satisfying.

\section{CONCLUSIONS}

This paper proposes an original way to approximate the non-separable prefilter of the hex-splines by different combinations of causal and anti-causal filters along the preferential directions of the hexagonal lattice. The design con- straint is imposed using powerful results of approximation theory. The obtained solutions are quasi-interpolants which exhibit the desired order of approximation and can be implemented completely in the spatial domain.

\section{REFERENCES}

[1] M. Unser, "Sampling-50 years after Shannon," Proceedings of the IEEE, vol. 88, no. 4, pp. 569-587, Apr. 2000.

[2] Dimitri Van De Ville, Rik Van de Walle, Wilfried Philips, and Ignace Lemahieu, "Image resampling between orthogonal and hexagonal lattices," in Proceedings of the IEEE International Conference on Image Processing, Rochester (NY), USA, Sept. 2002, vol. 3, pp. 389-392, IEEE.

[3] Carl de Boor, K. Höllig, and S. Riemenschneider, Box splines, vol. 98 of Applied Mathematical Sciences, Springer-Verlag, 1993.

[4] Eric Dubois, "The sampling and reconstruction of time-varying imagery with application in video systems," Proceedings of the IEEE, vol. 73, no. 4, pp. 502-522, Apr. 1985.

[5] Thierry Blu and Michael Unser, "Quantitative fourier analysis of approximation techniques: Part I-interpolators and projectors," IEEE Transactions on Signal Processing, vol. 47, no. 10, pp. 2783-2795, Oct. 1999.

[6] James C. Ehrhardt, "Hexagonal fast fourier transform with rectangular output," IEEE Transactions on Signal Processing, vol. 41, no. 3, pp. 1469-1472, Mar. 1993.

[7] A. M. Grigoryan, "Efficient algorithms for computing the 2-D hexagonal fourier transforms," IEEE Transactions on Signal Processing, vol. 50, no. 6, pp. 1438-1448, June 2002.

[8] Dimitri Van De Ville, Thierry Blu, Michael Unser, Wilfried Philips, Ignace Lemahieu, and Rik Van de Walle, "Hex-splines: a novel spline family for hexagonal lattices," IEEE Transactions on Image Processing, Submitted.

[9] M. Unser, "Fast B-spline transforms for continuous image representation and interpolation," IEEE Transactions on Pattern Analysis and Machine Intelligence, vol. 13, no. 3, pp. 277-285, Mar. 1991. 